\title{
Three around a Table: The Facilitator Role in a Co-located Interface for Social Competence Training of Children with Autism Spectrum Disorder
}

\author{
Massimo Zancanaro ${ }^{1}$, Leonardo Giusti ${ }^{1}$, Eynat $\mathrm{Gal}^{2}$, and Patrice T. Weiss ${ }^{2}$ \\ ${ }^{1}$ FBK, Trento, Italy \\ ${ }^{2}$ Haifa University, Haifa, Israel \\ \{zancana,giusti\}@fbk.eu, eynatgal@gmail.com, \\ tamararesearch.haifa.ac.il
}

\begin{abstract}
In this paper we describe a co-located interface on a tabletop device to support social competence training for children with Autism Spectrum Disorder. The interface has been developed on the multi-user DiamondTouch tabletop device as a 3-user application for two children and a facilitator (therapist or teacher). It takes advantage of the DiamondTouch table's unique ability to recognize multiple touches by different users in order to constrain interactions in a variety of ways. This paper focus on the support provided by the system to enhance a facilitator's management of interaction flow to increase its effectiveness during social competence training. We discuss the observations collected during a small field study where two therapists used the system for short sessions with 4 pairs of children. Although limited by the number of participants to date, the interactions that emerged during this study provide important insight regarding ways in which collaborative games can be used to teach social competence skills. Thus the children benefit from the motivational and engagement value of the games while the facilitator gains access to new tools to intrinsically support and shape the session.
\end{abstract}

Keywords: Autism Spectrum Disorder, collaborative games, multi-user colocated interfaces, Cognitive-Behavioral Therapy.

\section{Introduction}

Recent evidence indicates that collaborative computer games may be used to teach social competence $[9,11,12,15]$ and that tabletop technology is a promising tool for facilitating collaborative gaming experiences [22]. This approach has been successfully introduced as an educational tool for children with special needs due to the engaging and motivational effects of such computer games $[2,9,15,19]$ as well as the ability to adapt them to the requirements and limitations of children with special needs.

Autism Spectrum Disorders (ASD) is a neurological disorder that affects behaviour and the ability to communicate and interact socially. The extent of difficulties vary from one child to another but usually seriously affect their social interaction capabilities, the verbal and non-verbal communication and repertoire of activities and interests. Children with High Functioning ASD (HFA) have a normal or close to 
normal IQ, and some even exhibit exceptional skill or talent in specific areas. Still, dysfunction in social interaction and difficulties in emotional expression and recognition are indeed considered to be among the core deficits associated with ASD [1]. Training to improve social competence is therefore important for children with ASD, particularly for those with HFA [3].

When collaborative games are used in a therapeutic setting for children with HFA, the therapist plays a central role in facilitating tabletop activities and in leveraging the educational value of the experience. Yet, the design of these systems seldom explicitly acknowledges and empowers the role of the therapist as an active user of the interface. In this paper, we present the Join-In Suite, an application developed to train and enhance social competencies, specifically collaboration, of children with HFA using a therapeutic approach based on Cognitive-Behavioral Therapy (CBT).

In contrast to other work in the domain of technology for ASD, the Join-In Suite has been implemented on the DiamondTouch multi-user table [6] as a 3-user application for a therapist and two children. Three constraint patterns have been implemented as cooperative gestures on the interface; these are used extensively both to provide the therapist with specific controls to regulate the flow of interaction and to embed collaboration opportunities into the games.

The evidence collected during a formative evaluation indicated that the use of these constraint patterns effectively allows the therapist to pace the flow of activities and to shape the behavior of the children, promoting and regulating the collaboration experience.

\section{Related Work}

Despite the well-known benefits of using technology for children with ASD [11,20], as well as the benefits of CBT interventions for those with HFA [3], the CBT model has not yet been systematically implemented via technology.

Some encouraging results can be found in the literature on co-located applications for children with ASD. For example, Piper et al. [19] investigated how a four-player cooperative computer game that runs on tabletop technology was used to teach effective group work skills in a middle school social group therapy class of children with Asperger's Syndrome. Gal et al. [9] demonstrated the effectiveness of a threeweek intervention in which a co-located tabletop interface was used to facilitate collaboration and positive social interaction for children with HFASD; significant improvements in key positive social skills were achieved. Similarly, Battocchi et al. [2] studied the ability of a digital puzzle game to foster collaboration among children with low and high functioning ASD; in order to be moved, puzzle pieces had to be touched and dragged simultaneously by the two players.

Although all these systems assume that the facilitator (therapist or teacher) adopts the role of fostering and regulating the children's participation, this role has, to date, not been designed explicitly to be part of the interface.

Tabletop devices support user interaction in unique ways. Although accuracy of touch on a table top interface is inferior to mouse interaction [7], a touch modality appears to be preferable to a mouse and keyboard because it reduces the barrier 
between the user and the graphical elements of the interface. Hornecker and colleagues [8] showed that for collaborative tasks a direct touch interface is more effective than the use of multi-mice in particular for what concerns higher levels of awareness, fluidity of interactions and spatial memory. Direct manipulation becomes particularly useful for children who present different levels of motor coordination ability [19]. A further advantage of tabletop devices is that they can be made large enough to allow multiple users to collaborate without crowding; computer monitors are usually not big enough to allow equal visual perspective and interaction by more than one or two persons.

\section{Technology and Cognitive-Behavioral Therapy}

CBT is based on three assumptions: (1) interpersonal cognitive processes and emotions can mediate interpersonal behavior; (2) social problem solving and recognition of emotions can be taught cognitively and can influence behavior; and (3) social problem solving and a more comprehensive understanding of emotions can lead to later successful social adjustment. It also presumes that a more efficient cognitive understanding of the social world will lead to successful social adjustment in future situations.

There is good preliminary evidence of its potential for teaching social skills to children with ASD [3]. However, despite the well-known benefits of using technology for children with ASD [16], as well as the benefits of CBT interventions for those with HFA, there have been no attempts, thus far, to explore the ways in which CBT can be implemented via technology.

In a typical CBT session, a facilitator (either a teacher or a therapist with specific training) involves one or more children in some structured activities (the experience part) that allow the children to experience some social constructs which are then reflected upon (the learning part). The two parts are interleaved such that either may precede the other. The role of the facilitator is to form and expand the child's conceptual understanding of collaboration since only experiencing the task will not lead to inner understanding of the concept.

A technology designed to support a CBT session should therefore aim at three goals: (1) include the two parts: experience part to involve the children and learning part to help them to reflect; (2) support the facilitator to control activity flow and shape the collaboration experience; (3) embed specific interaction mechanisms to foster and promote collaboration between a pair of children.

\section{Join-In Suite}

The Join-In Suite was developed to address the area of social competence for children with HFA, and specifically their ability to collaborate with each other. It has been conceived as a 3-user application for a facilitator and two children and is intended to be analogous to a standard CBT session with the target population. 
The application has been designed by a team of interaction designers, computer scientists, educators and occupational therapists. The design cycles included two workshops with teachers, occupational therapists and children with HFA from two schools which offer programs to include children with ASD in mainstream education.

Following the tenets of CBT, the application is divided into a learning part which realizes a structured version of the CBT social problem solving technique and an experience part based on the CBT behavioral reinforcement technique. The former presents a series of social vignettes that present a social problem in which children select, suggest ways to solve the social problem and consider the possible consequences of each solution; the children then choose the best solution that will lead to a positive social experience. The latter consists of a game that allows the children to directly experience the chosen solution which is the social task to be acquired.

The application is implemented on the multi-user DiamonTouch device [6], a multi-user device which has the capability of recognizing different users when they interact with the system, i.e., it can track who is touching where. The Join-In Suite takes advantage of the DiamondTouch table's unique ability to recognize multiple touches by different users in order to constrain interactions in a variety of ways. For example, in some cases, to operate the system the children and the facilitator need to tap on the surface together (the system is not activated if the three of them don't touch the surface at specific places at the same time). In other cases, only the facilitator can activate certain functions (e.g., starting a game)

Furthermore, multi-user devices allow social rules to be "hard-wired" within the logic of the system which may be more effective than rules from a human facilitator. For example, the gestures of more than one user may be interpreted by the system to indicate a single, combined command or a "cooperative gesture" [17]; the latter can be used to increase the children's sense of teamwork and facilitate control on large, shared displays.

In our previous work [4], we focused primarily on Constraints on objects pattern: some elements of the system need to be operated by more than one user to be activated or used properly. We have now extended the available constraint patterns in the Join-In Suite to include: Different roles pattern: the individuals have to play different roles in order to perform a task (and the system prevents a single user from performing different roles at the same time) and Ownership pattern: participants have ownership of objects such that the system does not allow a user to use or access others' objects without explicit consent.

These three constraint patterns are used to foster collaboration between children. They also enhance the therapist's functionality by affording specific control over the interface elements, thereby providing opportunities to intervene in the flow of activity and to mediate the collaborative experience (see below).

\section{A Walk through the Interface}

The interface is oriented in such a way that both children sit on one side and the facilitator sits on the other side facing them. The facilitator controls the interface from 
a panel on one side of the table while the children interact with the surface from the other side (see figure 1).

The application provides access to several social stories (currently three) that exemplify problematic social situations. In order to choose a story, both the children and the therapist have to tap on the related card which displays a possible solution as a cartoon graphic. Each story has the same structure. During the learning part, the problem situation is presented together with five alternate solutions, one of which involves collaboration while the others offer selfish, non-collaborative or ineffective solutions to the problem; during the experience part, the children can play a game that represents the story. The facilitator can move between the two parts freely.

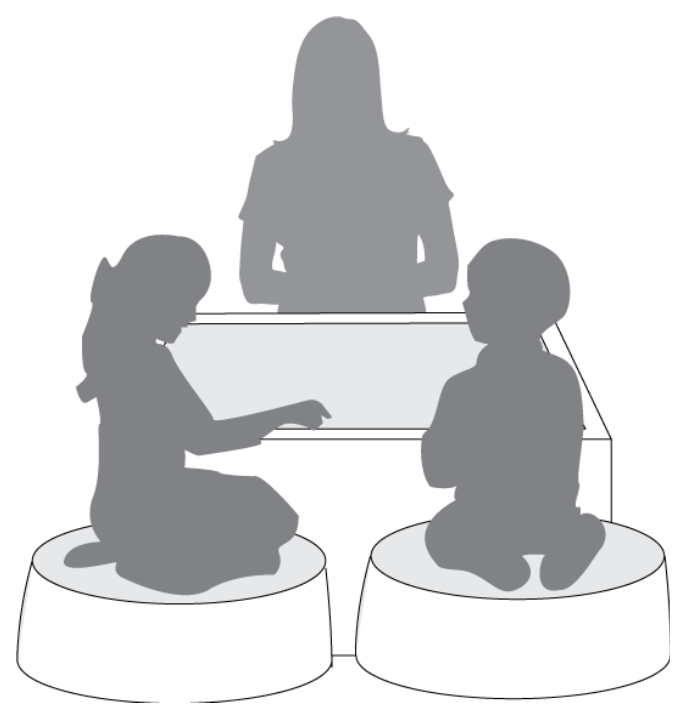

Fig. 1. Location of users during a Join In session

Figure 2 displays a screenshot of the learning part. The children can explore the different vignettes by selecting them and listening to an audio clip. Together with the facilitator, they can discuss possible solutions.

They then have to select one of the vignettes as the solution to the problem, by tapping simultaneously on it (the children and facilitator). If the selected alternative is not appropriate, the system provides a textual and auditory explanation and encourages the children to try something else. At any time during the activity, the therapist can decide to switch to the experience part (that is, the game).

\subsection{The Three Stories}

In the Apple Orchard story, the two children have to collect a number of apples for their mother to make jam but the basket is too heavy to carry individually. In the 
learning part, they are offered the alternatives of carrying the apples in their arms, asking their grandpa to carry it for them and other ineffective or non-collaborative strategies. They are also offered the "right" solution, that is, to carry the basket together.

The game implements the Constraints on Objects pattern and displays a basket that has to be moved to collect falling apples. The basket can be dragged by a single child but then it moves too slowly; when both children drag it simultaneously, it moves much more quickly so that the apples can be gathered before they fall to the ground.

The level of difficulty varies according to how the apples fall from the trees and can be controlled by the therapist; they can fall from lower or higher branches (and therefore the players have more or less time to collect them) and they fall down either one at a time or two together at different places so that the children need to negotiate which apple should be collected or lose both.

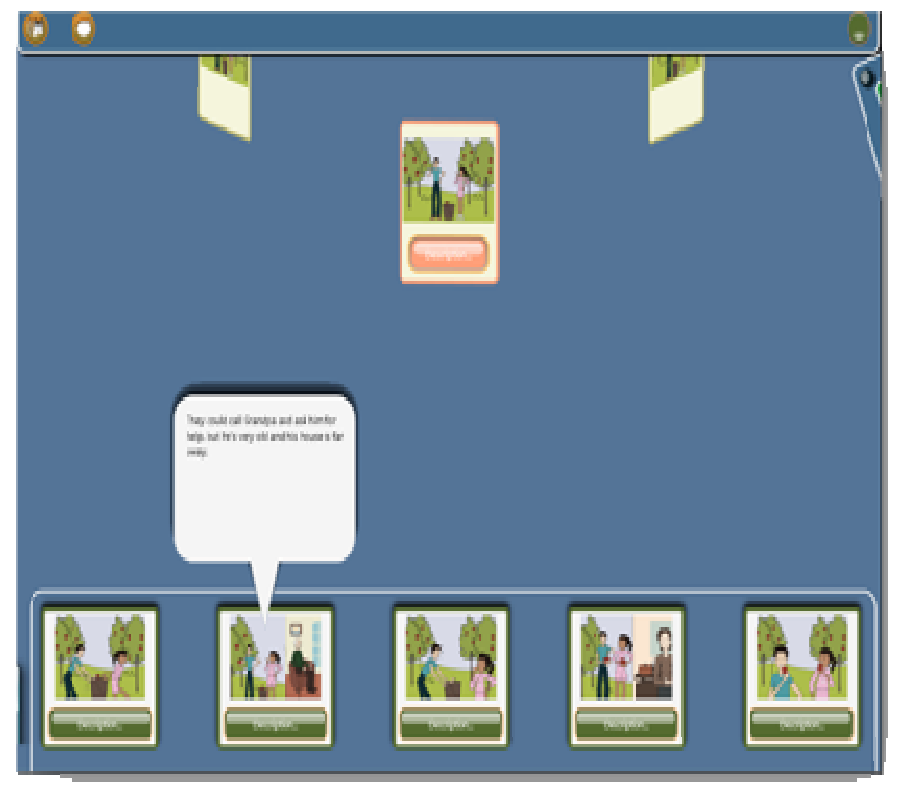

Fig. 2. A screenshot of the learning part of Apple Orchard

The Save the Alien story describes the situation of an alien starship that had a breakdown and made an emergency landing on Earth. The children are asked to help the starship by collecting shooting stars to be used as its fuel. Again, the children are offered 5 vignettes with 4 non-collaborative solutions and a collaborative one. 


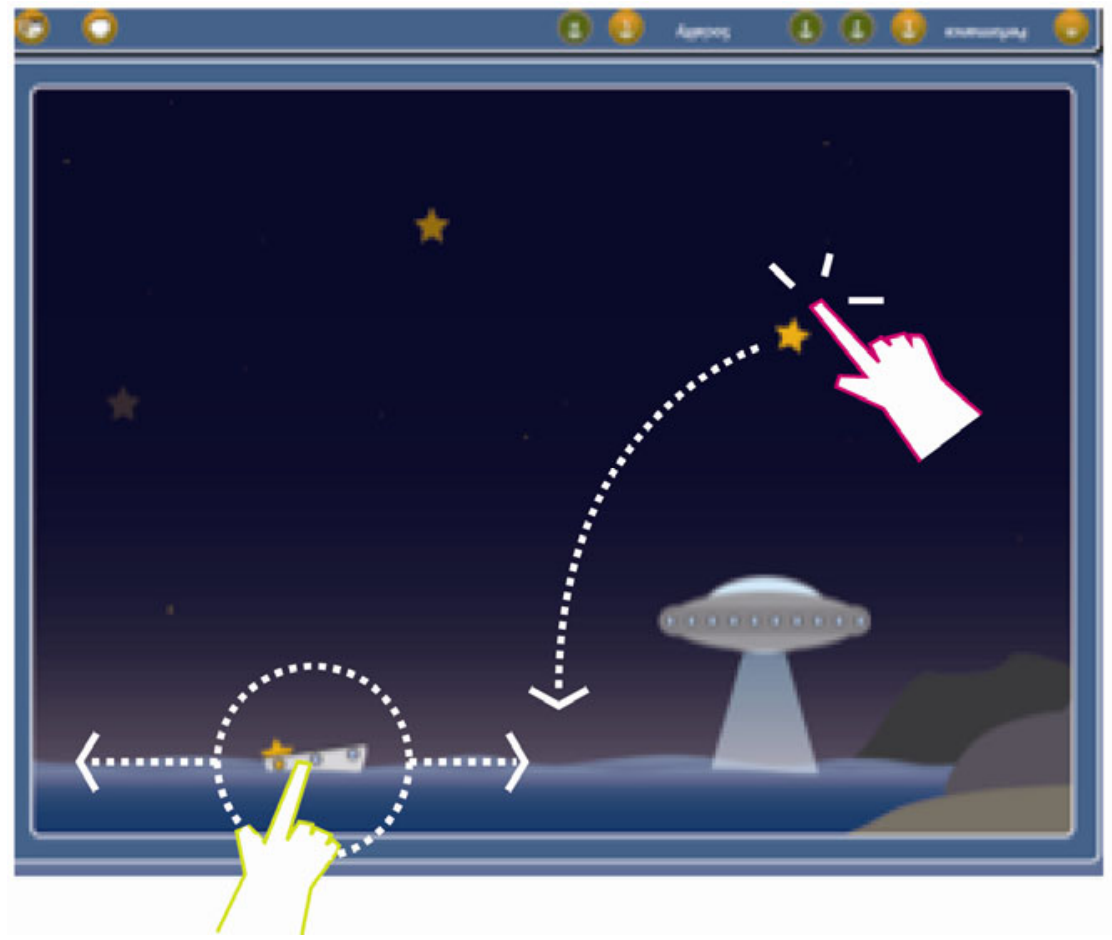

Fig. 3. A screenshot of Save the Alien game

The gaming part of the story implements the Different Roles pattern; one of the children has to catch the stars by tapping on them to make them fall toward the sea while the other child has to move a small boat to collect them (see Figure 3 ). The boat can accommodate only a small number of stars or it sinks; if a star falls into the sea, it causes waves that capsize the boat. The players have thus to carefully synchronize their actions in order to provide enough fuel (i.e., stars) to the starship before the time expires. The system prevents the child who drives the boat from also catching the stars and vice versa.

Finally, the Bridge story presents the situation of a fallen bridge that the two children have to rebuild. However, the pieces have been randomly strewn on the two banks of the river. The non-collaborative solutions include suggestions that one child can do all the work and the possibility of ignoring the problem while the "correct" solution is sharing each child's pieces that have fallen on the other bank of the other child.

The game part implements the Ownership pattern (see Figure 4). The system prevents the children from collecting pieces that are on the other's side of the river so they have to ask the other child to put it on a transport machine. 


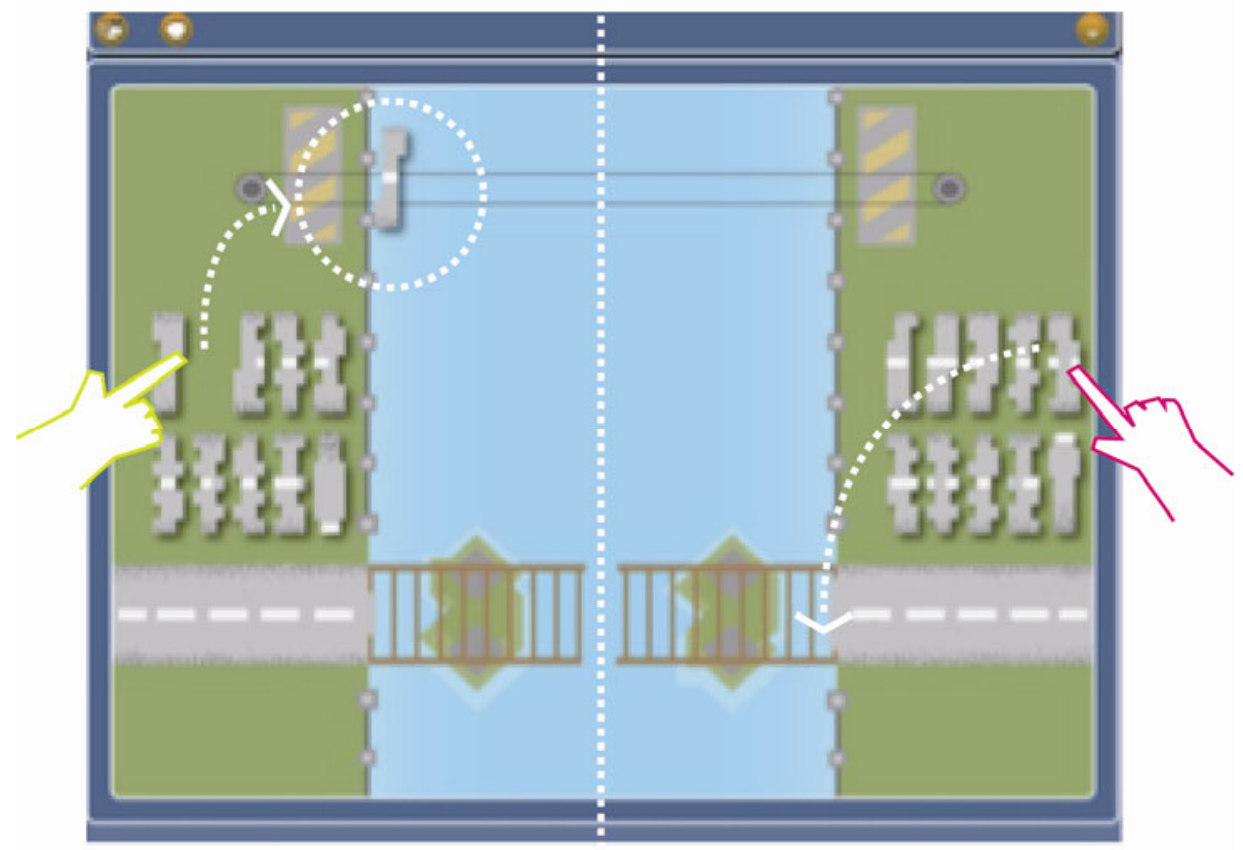

Fig. 4. A screenshot of the Bridge game

\subsection{The Facilitator as a Primary User of the Interface}

In designing a user interface it is important to clarify who are the primary users (those that actually perform actions on the interface) from the secondary users who do not use the interface directly although they are key actors in deciding the adoption of and the actual use of the system. In almost all educational and therapeutic systems, teachers and therapists are considered to be secondary users. In Join-In Suite, we have explicitly acknowledged their role as an additional primary user who interacts with the children using specific functions.

The constraint patterns introduced in the previous section have also been used in the design of the Join-In Suite to enable the facilitator to control the flow of interaction in the following manner: (1) some of the controls on the interface can be operated by the facilitator alone (Different Roles pattern); for example, the difficulty level of the game or the transition between the learning part and the game experience part (the same controls when operated by the children do not work); (2) some operations on the interface require a cooperative gesture (Constraints on Objects pattern) by all three users (the therapist and both children). In these cases the facilitator can refrain from contributing until there is more discussion by the children; (3) in each game, when a collaborative pattern is implemented, the facilitator can always act as a "super-user" (i.e., he can take either role in the Different Roles 
pattern, have access to any resource in an Ownership pattern and act as a replacement of either child in the Constraints of Objects pattern). In this way the facilitator can provide help to the children when they are not able to properly manage the games.

\section{Field Study}

A field study was conducted at a mainstream primary school that has three special classes for children with ASD. The evaluation involved 8 boys with HFA aged 9-12 years. All were enrolled in special education classes (Grades 2-5) within the elementary school. Two occupational therapists who work at the school were trained as facilitators to use the Join-In Suite application. Due to the large variability found amongst children with ASD, it is difficult to recruit a homogeneous group of children. Thus, studies on this population are usually made with smaller samples than in the case with some other user groups.

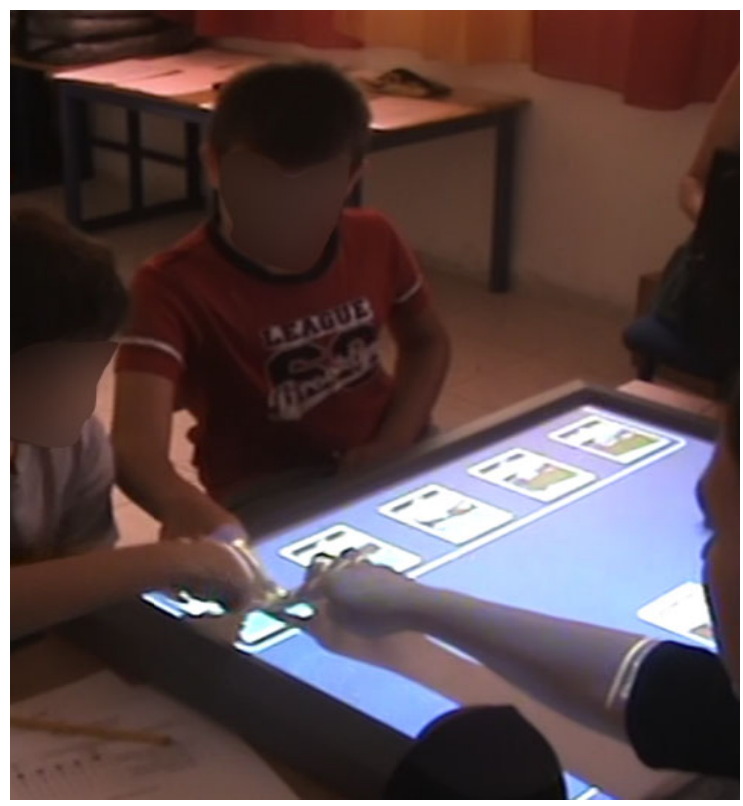

Fig. 5. Two children and the facilitator using the Join-In Suite

The study was organized as a qualitative/observational study to provide initial assessment of the application along several dimensions. We aimed primarily at collecting evidence of the impact of the system on the therapist/children interaction.

Each session was performed by one of two therapists and involved two children with HFA. First, the therapist introduced the Join-In Suite application to the children, who then were engaged in each of the three stories (Fig. 5). At the end of the session, the children were asked to rank the games and had a debriefing interview. 
All the sessions were video-recorded. Two video cameras were used; one facing the children to record all peer interactions, the other placed above the table to record the operations on the interface. At the end of the study, the two therapists were debriefed to discuss their experience with the application as a therapeutic tool.

\section{Results}

In this section, we briefly summarize the evidence collected on site and via videotaped observations and therapist de-briefing interviews. A qualitative analysis of the videotaped sessions and interview transcripts based on grounded theory was conducted. In this paper, the data are presented with a focus on the role of the facilitator; a general discussion on the dimensions of collaboration has been presented in [10].

\subsection{Facilitator Use of the System to Organize CBT Intervention}

Both the facilitators made use of the functionalities discussed in 5.2 to control the session. For example, during one of the sessions, one child was too dominant and the other child had to comply with his peer's commands. During the learning phase, the dominant child tapped which alternative was the most appropriate and "ordered" the passive child to touch the selected alternative. The more passive child simply followed this instruction; however, since the system also required the touch of the therapist to perform this action, she was able to use the triple-tap requirement (Constraints on Objects pattern) to limit and control the dominant child and involve the passive one. She purposely did not acknowledge the dominant child's choice and involved the passive child in a conversation which encouraged him to suggest an appropriate solution. In this case, the therapist used the triple-tap to shape the collaboration dynamics between the two children.

At another session, the facilitator used the triple-tap (Constraints on Objects pattern) to maintain control over activity flow. One of the children was hyperactive, impulsively touching many buttons and boxes on the table surface. In this case, the triple-tap was used to keep the activity at the discussion phase of the various alternatives until both children were ready to select the best solution. Rather than operating directly on collaboration dynamics, control was exerted over the pace of the activity.

In a third case, the facilitator used the possibility of being a super-user by helping the more passive child to move the boat in the Save the Alien game. In this way, she reduced the child's frustration but did not disrupt the flow of engagement of the other child. Note that game performance is not, in itself, important; rather it serves as a driver for the experience part. It is worth noting that when the facilitator acts a primary user the children's engagement was not reduced. On the contrary, the facilitator was often able to minimize a child's feelings of inadequacy due to poor performance that would otherwise divert his attention and make it more difficult for him to reflect on the collaboration task.

During the debriefing interviews the facilitators recognized the importance of these mechanisms in controlling the flow of interaction and suggested the addition of 
further ways to achieve explicit control. In particular, they suggested that the system was too structured in forcing a sequence of activities for the learning part while they sometime felt the need, for example, to temporarily remove the alternatives in order to focus on the story again. Furthermore, they recommended the addition of a general pause button that can be operated by the facilitator if the children become too distracted or engage in repetitive behaviors typical of ASD.

\subsection{Interleaving the Experiential Part in the Form of Games with Learning Objectives and the Learning Part}

A major factor that distinguishes Join-In Suite from commercial computer and video games is the therapeutic model behind it. Indeed, the children were always very focused during the games, but they tended, as expected, to be somewhat distracted during the learning part. The ability to interleave learning and gaming was very effective to maintain the coherence and engagement of the whole experience.

The two facilitators used, in general, two different approaches: one preferred to go from learning to experience in order to better explain the concept of cooperation while the other sometimes went from experience to learning to introduce the concept of cooperation in a more natural manner via the game rules. Both approaches were effective and it will be important to observe the strategies used by other facilitators over a longer period of time to understand and expand upon the system's flexibility in adapting to different approaches to manage the session.

The facilitators appreciated that the multimedia cards were more engaging for the children than the conventional, paper-based vignettes they were used to. Still, they also expressed a concern that the system only offered exploration of a predetermined set of cards. Indeed, they did involve the children in discussions aimed at proposing their own solutions but these discussions could not be incorporated into the interface in the form used during the field study.

\subsection{Using Game Structure to Balance Role of the Facilitator}

As discussed above, the constraint patterns may be used to provide ways for a facilitator to exercise control over the interaction flow. Yet, it is also important that the facilitator be able to "fade out" in order to foster direct interaction between the children. In general, our observations confirm the findings of Piper and colleagues [19] about the motivational and engaging roles of constraint patterns embedded in the system for these types of users. All the games were engaging even if several factors made the Apple Orchard less successful than Bridge and Save the Alien (i.e., the basket was difficult to grab together and collecting falling apples was not considered to be an exciting task by the children). (In fact, differences in how the children viewed the games were a positive factor since it allowed them to feel and affirm an element of choice.)

The three games elicited different collaborative strategies. The Bridge, which of the three games was the most appreciated by the children, was very effective in eliciting a negotiation dialogue centered on the notion of sharing. During one session, one child who has limited visual matching abilities was helped by his peer who was more adept at identifying the correct puzzle pieces. He explicitly asked for help 
during the game. During the final interview, the child said: "In the bridge I learned that sometimes there is a need for another person to help on the other side". The Save the Alien game elicited mainly dialogue aimed at achieving real time motor coordination to synchronize actions such as "Now, I'm hitting a star, are you ready?" The constraints imposed by the system (a child cannot do both tasks) led naturally to this kind of interaction without the need of the facilitator to determine and control the roles. These aspects were positively recognized by the therapists who supported our view that verbalization of collaborative behaviors is a fundamental therapeutic issue.

As discussed above, in some cases the facilitators decided to intervene in the game to support one the children whose game performance was not adequate. They further suggested the possibility of directly controlling the duration of the game. Though the pressure of a time-limit for a game is an important aspect for the engagement, sometimes it was frustrating for the children to fail the game because they were too slow. The facilitator is now able to extend time simply by tapping on the clock.

\section{Redesign of the Interface}

The insights of the formative evaluation were used to refine the design of the Join-In Suite (Fig. 6). For what concerns the role of the facilitator as a primary user, two areas of improvement were identified.

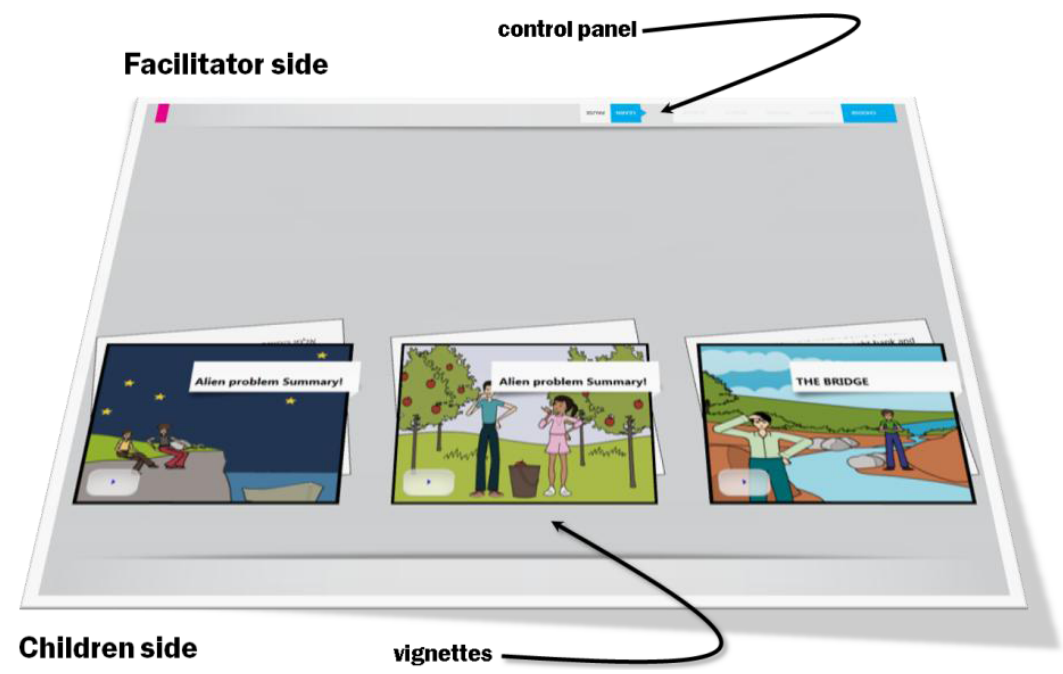

Fig. 6. A screenshot of the new interface of Join-In Suite

First, we provided a more effective way of controlling the activity, in particular in enabling the facilitator to move back and forth to the different sub-tasks of the learning part and between the learning and the game parts. Second, we enabled the facilitator to involve the children in proposing alternative solutions rather than just discussing the ones previously determined by the system. 
This redesign mainly consisted of making the sub-tasks of the learning activity more easily identifiable and navigable on the interface. We identified 5 steps in the learning activity that are now presented as explicit steps in the new interface (including the new step for the recording of an alternative proposed by children): (1) choose step where the social story is chosen amongst the three available; (2) present step is where the social story is introduced as a social problem and discussed by the children and the facilitator; (3) record step where the children can record their own solution to the social problem; this step is optional and the facilitator can skip or postpone it (4) select step where the children are asked to consider the 4 different alternatives provided by the system (and if present the one recorded by the children) to choose the most adaptive one which best solves the presented social problem; and finally (5) the sum up step where the social problem and the chosen solution are presented together and the facilitator can take the opportunity for further elaboration; this step is also optional.

\subsection{The Control Panel}

The facilitator control panel has been completely redesigned and is now a tool to control the system and as a navigational aid to access the 5 steps and move between the two learning and experience parts. As in the previous version, we exploit the multi-user capabilities of DiamondTouch to prevent the children from operating on the toolbar.

The new control panel is displayed in Figure 7. Two buttons positioned at the center of the panel enable switching between the learning and game parts. When the Learn button is selected, the step selector is visible on left side. When the Play button is selected the step selector is made invisible and a new panel appears on the left with some game specific controllers (see below).
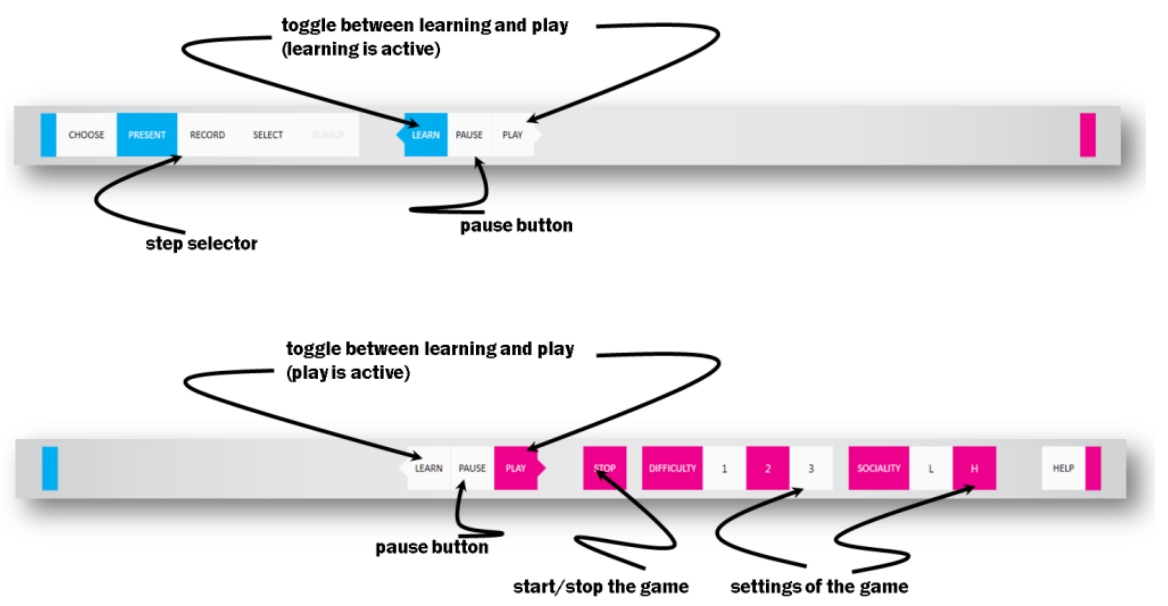

Fig. 7. Facilitator's new control panel: on the top there is the Learn configuration and on the bottom the Play configuration 
In the Learn configuration, a step selector is displayed that lists the 5 steps as buttons. In order to move to a specific step, the facilitator presses the corresponding button. The selected button signals the current step. When a step cannot be accessed, the corresponding button is disabled, that is the corresponding button is displayed with a light gray label and cannot be selected (for example, before the completion of the choose step, i.e., before selection of the social story, the other steps cannot be accessed).

In the Play configuration, the game settings (level of difficulty of the game and required level of interaction between the children) can be adjusted. The game is started and stopped with the appropriate buttons.

Positioned between the Learn and Play buttons, there is a Pause button. It can be used in any time to freeze the application. When this button is selected, nothing can be done on the interface. To continue using the application, only facilitator can press again the same button.

\subsection{The Record Card}

When entering in the record step, a small control panel for managing the recording appears below the corresponding step button in the control panel step selector (Figure 8). Again, only the facilitator can use this button to record the children narrating their own solution.

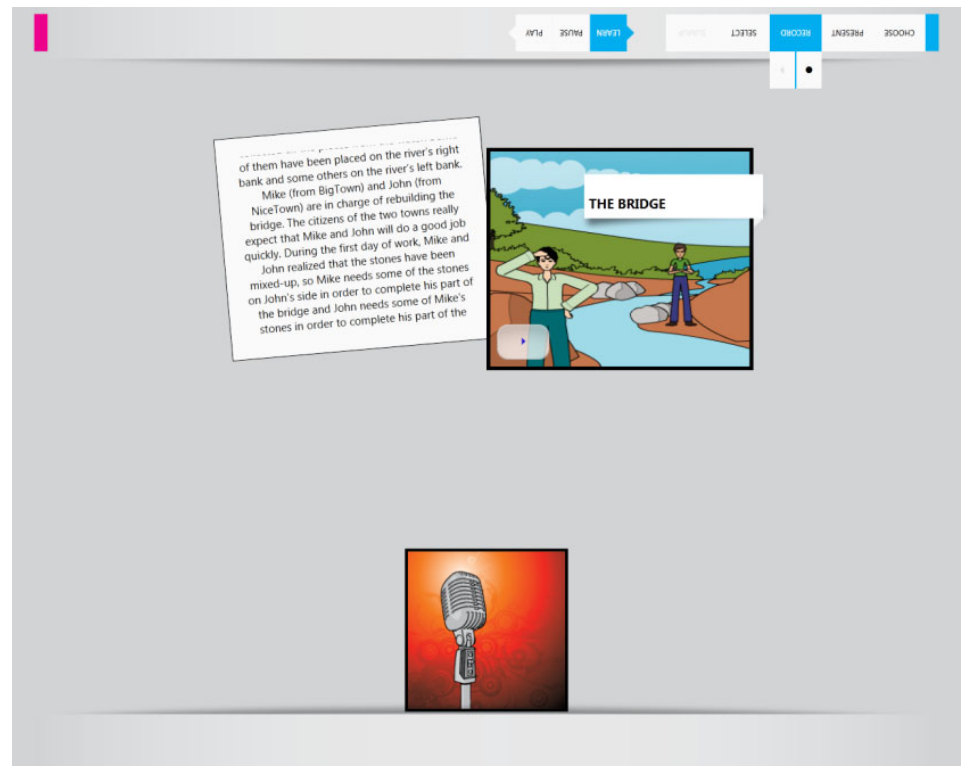

Fig. 8. A screenshot of the Record step in Join-In Suite

The system displays a card that is then shown together with the system alternatives in the Select step. This card can be recorded as many times as needed but a new recording cancel the previous ones (though all the recordings are stored by the system 
for logging and analysis). Once the card has been recorded, the facilitator can decide to move to the next step; the card will be shown together with the system generated alternatives.

\section{Discussion}

This initial formative evaluation of the Join-In Suite provided valuable insight regarding the possibility of using collaborative games as a basis for teaching social competence skills. Join-In Suite's strengths derive not only from the motivational and engagement value of the learning and gaming tasks but also from the provision of new tools that intrinsically support a facilitator while conducting a session.

A number of studies have demonstrated the ability of children to acquire skills when interacting independently with computer technology $[21,18]$. The capacity of software programs to deliver stimuli, reinforce correct responses, and demonstrate errors, all in a reliable, controlled and motivating manner, make this medium attractive as an educational and therapeutic tool. In addition, such programs may enable educational interventions to be delivered by staff and family members who have not had explicit training.

Much has been written about how to design software to help children with ASD improve in their academic, social and communication skills [18]. Davis et al [5] and Jordan [12] recommend that specific factors should be taken into account when designing learning environments for children with ASD in order to enhance their strengths while reducing the need for abilities which are more difficult for them. First, it is important that both the task and the actions of the facilitator be reliable, consistent and predictable. Second, the introduction of novel elements must be done in a gradual and controlled manner. Third, the computer-based learning activities should be challenging, but children with ASD should not be overly penalized for mistakes. It must be recognized that while experiencing a virtual environment they may apply strategies that helped them in the past in a real world, setting but do not work as well in a computer-based environment. Thus it is best when negative feedback is provided together with clear cues as to how to proceed. Fourth, it is important to use time as a motivating factor but not in a way that will add pressure to the task and that will not permit sufficient practice to achieve mastery. The Join-In Suite has explicitly incorporated these design guidelines and, as demonstrated by both the focus groups and formative studies, they have proven to be appreciated by teachers, therapists and children with ASD as we discussed in greater depth in [10].

Far less attention has been paid in the literature to the role of the facilitator as an active user of the computer game. Although there is an increasing amount of evidence about the beneficial effect of the presence of a facilitator in educational interventions for children with ASD (e.g., [14]), for most educational games, the facilitator acts as a secondary user by moderating access to the game but without actually using the interface. In Join-In, because the interface has been designed to give the facilitators a fundamental and dynamic role in shaping the children's experience, they become primary users who directly operate on the interface. They set the pace of the activity, influence the dynamics between the children, and help them reach a reasonable level of performance. Just as a therapist acts as a moderator during 
a conventional CBT session (controlling the pace, supporting the performance of activities, encouraging reflection), Join-In acknowledges this role and provides explicit support to achieve it.

The type of functionalities offered to the facilitator can be broadly classified into two categories: those that can be used to limit non-adaptive behavior by the children and those that may be used to empower them.

ASD is a very broad classification that includes different levels and types of social difficulties. With Join-In, we include a sophisticated way of reducing the impact of social avoidance behavior and, at the same time, enhance the motivation to communicate with the other. These are mainly achieved by the need to explicitly recognize the contribution of the peer (and the facilitator) in order to operate the interface. In addition, the facilitator, as a primary user, may exert this controlling effect when supported by appropriate constraints on the interface; the addition of a pause button for the facilitators allows them to freeze the application at any time providing further control of non adaptive behaviors of one or both children.

In contrast, it is well known that social avoidance behavior in children with ASD may be reduced when they are involved in child-preferred activities [13]. Allowing the children a degree of flexibility in choosing and organizing their preferred activities, may empower them and lead to more engagement in the task. It is important to note that the facilitators in our study were able to use the interface to exert this control and to engage children. They also asked for more explicit controls to empower the children, for example, the possibility of extending the time of game.

Requiring the involvement of 3 players, one of them being a moderator, Join-In provides a strong assistive tool to the teacher, rather than replacing her. Our experience has shown that trained therapists were able to rapidly learn how to use Join In to implement CBT-based social competence training. The Suite appears to be sufficiently usable that even less experienced operators could use it to provide additional training opportunities to the children.

\section{Conclusion}

In the paper, we presented a tabletop interface based on a multi-user device to support teaching social competence skills in children with ASD. This paper focused on the role of the facilitator as a primary user of the interface and how the functionalities provided by the system enhance the management of interaction flow and increase its effectiveness. In particular, two broad categories of functionalities are offered to the facilitator: those that may be used to limit the negative behaviors of the children and those that may be used to empower them.

Our experience has shown that trained therapists were able to rapidly learn how to use Join-In to implement CBT-based social competence training. Although limited by the number of participants to date, the interactions that emerged during this study provide important insight regarding ways in which collaborative games can be used to teach social competence skills. In particular, the use of the three constraint patterns in the design of the interface enabled the facilitator to effectively pace the flow of activities and to shape the children's behavior, promoting and regulating the collaboration 
experience. We do not claim that these three patterns are sufficient for these types of interaction; rather, they demonstrate a proof of concept as a starting point for exploring further implementations and additional patterns.

Acknowledgments. This work was partially funded by the European project COSPATIAL (FP VII - 231266). We thank Nirit Bauminger for her key contributions to the application of the CBT model and to the design phase of the Join-In Suite; Sigal Eden for her help in the preparation of the formative study; Alberto Battocchi for the initial concept of Join-In and Francesco Telch for the implementation of the prototype. Finally, we want also to thank the children and the therapists who participated in the study.

\section{References}

1. American Psychiatric Association, Diagnostic and statistical manual of mental disorders, 4th edn., text revision, Washington, DC (2000)

2. Battocchi, A., Ben-Sasson, A., Esposito, G., Gal, E., Pianesi, F., Tomasini, D., Venuti, P., Weiss, P.L., Zancanaro, M.: Collaborative Puzzle Game: a Tabletop Interface for Fostering Collaborative Skills in Children with Autism Spectrum Disorders. Journal of Assistive Technologies, 4(1), 4, 14 (2010)

3. Bauminger, N.: Group social-multimodal intervention for HFASD. Journal of Autism and Developmental Disorders 37(8), 1605-1615 (2007)

4. Bauminger, N., Gal, Y., Goren-Bar, D., Kupersmitt, J., Pianesi, F., Stock, O., Weiss, P.T., Yifat, R., Zancanaro, M.: Enhancing Social Communication in High Functioning Children with Autism through a Colocated Interface. In: Proceedings of IEEE Multimedia Signal Processing 2007 - Special Session on Multimedia Signal Processing for Education Applications, Chania, Greece (2007)

5. Davis, M., Dautenhahn, K., Powell, S.D., Nehaniv, C.L.: Guidelines for Researchers and Practitioners Designing Software and Software Trials for Children with Autism. Journal of Assistive Technologies 4(1), 38-48 (2010)

6. Dietz, P.H., Leigh, D.L.: DiamondTouch: A Multi-User Touch Technology. In: Proceedings of User Interface Software and Technology (UIST), Orlando, FL (2001)

7. Forlines, C., Wigdor, D., Shen, C., Balakrishnan, R.: Direct-Touch vs. Mice Input for Tabletop Displays. In: Proceedings of ACM Computer Human Interaction, CHI 2007, pp. 647-656 (2007)

8. Hornecker, E., Marshall, P., Sheep Dalton, N., Rogers, Y.: Collaboration and Interference: Awareness with Mice or Touch Input. In: Proceedings of ACM CSCW 2008, pp. 167-176 (2008)

9. Gal, E., Bauminger, N., Goren-Bar, D., Pianesi, F., Stock, O., Zancanaro, M., Weiss, P.L.: Enhancing social communication of children with high functioning autism through a colocated interface. Artificial Intelligence \& Society 24, 75-84 (2009)

10. Giusti, L., Zancanaro, M., Gal, E., Weiss, P.L.: Dimensions of Collaboration on a Tabletop Interface for Children with Autism Spectrum Disorder. In: Proceedings of ACM Computer Human Interaction, CHI 2011 (2011)

11. Grynszpan, O., Martin, J.C., Nadel, J.: Designing Educational Software Dedicated to People with Autism. In: Proceedings of AAATE 2005, Lille, France, vol. (2005) 
12. Jordan, R. (1997) Education of Children and Young People with Autism, Guides for Special Education no 10

http: / / unesdoc . unesco.org/images / 0011/001120/112089eo.pdf (accessed January 2011)

13. Koegel, R.L., Dyer, K., Bell, L.: The influence of child-preferred activities on the children's social behavior. Journal of Applied Behavioral Analysis 20, 243-252 (1987)

14. Kroeger, K.A., Schultz, J.R., Newsom, C.: A Comparison of Two Group-Delivered Social Skills Programs for Young Children with Autism. Journal of Autism Dev. Disord. 37, 808-817 (2007)

15. Martinez, R., Kay, J., Yacef, K.: Collaborative Concept Mapping at the Tabletop. In: Proceedings of ACM International Conference on Interactive Tabletops and Surfaces (2010)

16. Moore, D., McGrath, P., Thorpe, J.: Computer-aided learning for people with autism-a framework for research and development. Innovations in Education and Training International 37(3), 218-228 (2000)

17. Morris, R.M., Huang, A., Paepcke, A., Winograd, T.: Cooperative Gestures: Multi-User Gestural Interactions for Co-located Groupware. In: Proceedings of ACM Computer Human Interaction CHI 2006, Montréal, Québec, Canada (2006)

18. Pennington, R.: Computer-Assisted Instruction for Teaching Academic Skills to Students With Autism Spectrum, Focus Autism Other Dev. Disabl. (August 2010)

19. Piper, A.M., O’Brien, E., Morris, M.R., Winograd, T.: SIDES: A Cooperative Tabletop Computer Game for Social Skills Development. In: Proceedings of CSCW (2006)

20. Putnam, C., Chong, L.: Software and technologies designed for people with autism: what do users want? In: Proceedings of the 10th International ACM SIGACCESS Conference on Computers and Accessibility (2008)

21. Roschelle, J., Pea, R., Hoadley, C., Gordin, D., Means, B.: Changing How and What Children Learn in School with Computer-Based Technologies. In: The Future of Children, vol. 10(2), pp. 76-101. Packard Foundation, Los Altos (2001)

22. Tse, E., Greenberg, S., Shen, C., Forlines, C.: Multimodal multiplayer tabletop gaming. Comput. Entertain. 5(2), 12 (2007) 